\title{
Computational Imaging at Microwaves using Compressive Sensing
}

\author{
Benjamin Fuchs, Ariel Christopher Tondo Yoya, Mor Diama Lo and Matthieu Davy \\ Institute of Electronics and Telecommunications of Rennes, UMR CNRS 6164, University of Rennes 1, France \\ e-mail: \{benjamin.fuchs;matthieu.davy\}@univ-rennes1.fr
}

Imaging systems in the microwave range aim to reconstruct the spatial distribution of the scattering strength of a scene. In conventional systems, several antennas are used in emission and / or reception, thanks to either a physical antenna array or a virtual one by moving an antenna (synthetic aperture radar). An image of the scene can then be constructed by combining coherently the signals recorded by the antennas. Recently, computational imaging has received a lot of attention. Instead of measuring the field on an array of sensors, the spatial information of a scene is encoded onto independent illuminations collected by a single sensor, paving the way to the design of cheap and fast imaging devices.

In microwaves, highly dispersive medium such as metamaterial apertures [1], frequency diverse metasurfaces [2] and leaky chaotic cavities [3,4] (our choice) are efficient hardware sensing devices to achieve computational imaging. More specifically, the reverberation of the wave propagation inside the chaotic cavity emulates random illuminations of a scene at different frequencies. Computational imaging requires to first compute the transfer matrix frequency/space $\mathbf{A}(\omega, r)$ that links the sensor and the discretized volume of the scene for each illumination. This step can be achieved by a scan in the near field of the leaky cavity and a projection of this scan on the scene. Once this calibration step done, an image can be built from the signal $\boldsymbol{y}(\omega)$ recorded with only a single (or two) receiving antennas by solving an inverse problem that can be formulated in the following generic way:

$$
\min \|\boldsymbol{\sigma}(r)\|_{p} \text { subject to }\|\boldsymbol{y}(\omega)-\mathbf{A}(\omega, \mathrm{r}) \boldsymbol{\sigma}(r)\|_{2} \leq \delta
$$

where $\boldsymbol{\sigma}(r)$ is the scattering strength of the scene to be reconstructed and the parameter $\delta$ is a positive scalar to take into account inevitable additive noise mainly due to the measurement uncertainties of $\mathbf{A}(\omega, \mathrm{r})$. The resolution of the reconstructed images can be enhanced by leveraging the sparsity inherent to most scenes. The $l_{l}$ norm ( $p=1$ in equation (1)), that is known to promote sparse solutions, is chosen to regularize the solution. The proposed imaging system turns out to be an effective compressive imaging device which exploits the natural randomness of the matrix $\mathbf{A}(\omega, \mathrm{r})$.

A leaky chaotic cavity of outer dimensions $50 \times 50 \times 30 \mathrm{~cm}^{3}$ has been manufactured at IETR. The cavity is made chaotic by adding inside three metal hemispheres on the walls and a deformed corner. Two coaxial to waveguide transitions are connected to the sides of the cavity to emit/record the signal in X-band (8-12GHz). Some preliminary experimental results are shown in Fig. 1.

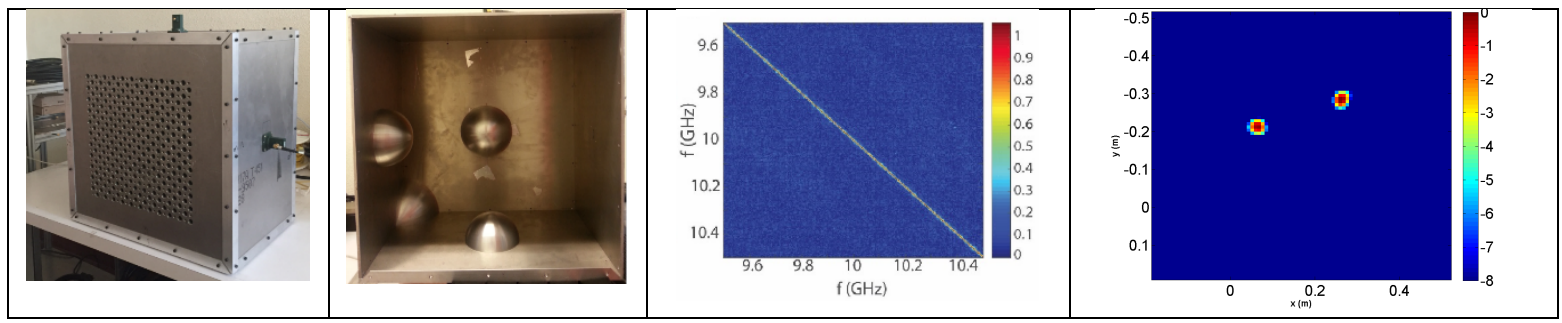

Figure 1. From left to right: manufactured leaky chaotic cavity (outer and inside views), spectral field correlation matrix of the cavity and reconstruction of two ponctual targets using a sparsity regularization.

1. J. Hunt, T. Driscoll, A. Mrozack, G. Lipworth, M. Reynolds, D. Brady, and D. R. Smith, "Metamaterial apertures for computational imaging," Science, 339(6117):310-313, 2013.

2. J. Gollub et al., "Large metasurface aperture for millimeter wave computational imaging at the humanscale,"Sci. Rep., vol. 7, p. 42650, Feb. 2017.

3. T. Fromenteze, O. Yurduseven, M. F. Imani, J. Gollub, C. Decroze, D. Carsenat, and D. R. Smith. "Computational imaging using a mode-mixing cavity at microwave frequencies." Appl. Phys. Lett. 106, 194104, 2015.

4. C. Yoya Tondo, B. Fuchs, M. Davy, "Computational passive imaging of thermal sources with a leaky chaotic cavity,” Appl. Phys. Lett. 111, 193501, 2017. 